\title{
Innovación y gestión de la identidad en tiendas de abarrotes en México y Colombia
}

\section{Innovation and Identity Management in Grocery Stores in Mexico and Colombia}

Jorge William Tenjo Beltrán ${ }^{\star}$

Universidad Anáhuac México

Av. Universidad Anáhuac núm. 46, Col. Lomas Anáhuac,

C.P. 52786, Huixquilucan, Estado de México

Editor: Rogelio del Prado Flores williamtenjo@outlook.com

https://orcid.org/0000-0001-5282-0615

https://doi.org/10.36105/stx.2019n3.05

\section{RESUMEN}

- 1 objeto de este trabajo es mostrar la situación de las tiendas de abarrotes como microorganizaciones por medio de la innovación y analizar la presencia de dicha capacidad desarrollada entre las mipymes junto a la importancia de la gestión de la identidad en la comunicación organizacional para apalancar acciones de mejoramiento. El trabajo se dimensiona y desarrolla a través de un comparativo en las diferentes comunidades de dos países como México y Colombia representadas en un territorio local en este tipo de negocios. Permitiendo promover nuevas perspectivas de la comunicación organizacional para las microorganizaciones.

Palabras clave: comunicación organizacional, innovación, identidad, mipymes, microorganizaciones.

\section{ABSTRACT}

The purpose of this work is to show the situation of grocery stores as microorganisms through innovation and analyze the presence of such capacity developed among MSMEs together with the importance of identity management in organizational communication to leverage

\footnotetext{
* Maestro en Mercadotecnia y Publicidad por la Universidad Anáhuac México; estudiante de tiempo completo del Doctorado de Investigación de la Comunicación (PNPC) en la Universidad Anáhuac México.
} 
actions of improvement. The work is sized and developed through a comparison in the different communities of two countries such as Mexico and Colombia represented in a local territory in this type of business. Allowing to promote new perspectives of organizational communication for microorganisms.

Keywords: organizational communication, innovation, identity, MSMEs, microorganizations.

\section{INTRODUCCIÓN: LAS MIPYMES EN MÉXICO Y COLOMBIA}

Han sido recurrentes las comparaciones entre México y Colombia en varios aspectos, como la indudable similitud producto de la herencia hispanoamericana y del desarrollo histórico como países independientes en la consolidación de Estados soberanos. Sin embargo, el más relevante para este trabajo son las estrategias semejantes de vinculación con la economía internacional a través de la liberación de sus economías y una extensa apertura comercial.

México, un país formado por una cifra aproximada de 119 millones 530 mil 753 habitantes según la encuesta Intercensal 2015, realizada por el INEGI, cuenta con 7,236,821 micro negocios, 6,178,906 pequeños establecimientos, 4,293,422 medianos establecimientos, 3,610,825 grandes establecimientos, 2,193,087 establecimientos relacionados con el gobierno, $2,145,805$ otro tipo de establecimientos y 864,606 no especificados. Hay que entender que además que, las pymes surgieron como fuente para suministrar el consumo de los habitantes en las pequeñas poblaciones, este tipo de establecimientos en México ayudan a la economía dando empleo a casi $72 \%$ de la población y contribuyendo aproximadamente con $52 \%$ del Producto Interno Bruto (PIB). A esto se añade que, en México, la clasificación empresarial se encuentra reglamentada en la Ley para el Desarrollo de la Competitividad de la micro, pequeña y mediana empresa del año 2000, que tiene por objeto promover el desarrollo económico nacional a través del fomento de las creaciones de este tipo de empresas y el apoyo para su viabilidad, productividad, competitividad y sustentabilidad.

Por otro lado, en Colombia, un país formado por una cifra aproximada de 48 millones 574 mil 840 habitantes, cuenta con una estructura empresarial, conformada principalmente por micros y pequeñas empresas, con capital familiar; de esta forma, las pymes según el Ministerio de Economía, Industria y Comercio están delimitadas en razón del número de empleados que, en este caso, se toman entre 11 y 199 personas y un valor de activos entre 45 mil a 1 millón de dólares (FUNDES, 2008). E1 95\% del parque empresarial corresponde 
a pymes, de las cuales, por tamaño $74 \%$ son micro, $12 \%$ pequeñas y $14 \%$ medianas. Por sector, $83 \%$ corresponden a servicios y comercio. A esto se añade, en el cual la forma de empleo, que las pymes llegan a concentrar $48 \%$ de todo lo que genera el sector privado, donde las agrícolas llegan a aportar $20 \%$. Además, por sector, $39 \%$ de puestos generados corresponden a servicios, $32 \%$ al comercio, $22 \%$ a la industria y $7 \%$ a las TIC. De acuerdo con lo enunciado anteriormente podemos conjeturar que, las micro, pequeñas y medianas empresas de Colombia llegan a tener un peso de 30\% en el Producto Interno Bruto (PIB) siendo fundamental para la generación de empleo. También se han convertido en la gran apuesta para alcanzar la internacionalización de la economía y las tasas de crecimiento que requiere el país para reducir la pobreza.

\section{LA IMPORTANCIA DE LAS MIPYMES DE ABARROTES}

\section{EN LA ECONOMÍA DE MÉXICO Y COLOMBIA}

En los últimos años se puede constatar que en los dos países hay muy pocas investigaciones en el campo académico donde traten el tema de las tiendas de abarrotes o de barrio con respecto a todas sus características. De acuerdo con el Directorio Estadístico Nacional de Unidades Económicas del INEGI 2015, en México hay 975,760 negocios de comercialización de abarrotes al menudeo, sobresalieron con respecto al total del personal ocupado, 90.0\% (la mayoría dueños y familiares). Según Nielsen en Colombia, el número de establecimientos del sector comercial corresponde a 307,000 a tiendas de barrio (Dinero, 2014).

Las dificultades que enfrentan las tiendas de abarrotes en México es la competencia a escala ampliada de las tiendas de autoservicio, tipo Walmart o Soriana, y de las tiendas de conveniencia, tipo Oxxo y Seven Eleven con una expansión acelerada, creando una disputa monopólica convirtiéndose en un riesgo para muchas tiendas. A pesar de los obstáculos en estos establecimientos, la tendencia devolvió al canal tradicional el liderazgo en el mercado, al aumentar en $37.9 \%$ en el 2014. Así como el crecimiento del sector comercio en la venta específica de abarrotes representó $74.8 \%$ y en el lapso de referencia el incremento fue de 4.3 por ciento (El Economista, 2016). Por otra parte, la disminución en el ingreso disponible de los mexicanos, como consecuencia de mayores impuestos, ocasionó que los consumidores comprarán más en las — tienditas de la esquina — como son conocidas coloquialmente, que en las grandes empresas que acaparan el mercado del comercio de alimentos. Hay que entender que el comercio de abarrotes cumple un papel fundamental para la distribución y venta (es decir, realización financiera) de varias de las grandes empresas de este país: Bimbo, Gamesa, refresqueras, cerveceras, chicleras, dulceras, Sabritas, Procter y Gamble, etcétera. 
Ninguna de esas grandes empresas existiría sin la enorme red de distribución económica que suponen las tienditas de la esquina. La existencia de estas tiendas es un canal de distribución insustituible para estos negocios que en su mayoría corresponde a transnacionales (Martin, 2017).

De otro lado, el panorama en Colombia del comercio de abarrotes y la compra en la tienda de abarrotes sigue reinando, pese a que los grandes supermercados, hipermercados, tiendas de conveniencia y grandes empresas como Almacenes Éxito mantuvieron un cómodo liderazgo, mientras que Supertiendas Olímpica ascendió al segundo puesto al superar a Cencosud Colombia (Jumbo). Posteriormente se posicionaron Alkosto, Almacenes La 14, Super Inter Supermercados, Makro Super Mayorista, y Tiendas D1, donde algunas han incursionado con fuerza en los barrios del país con formatos de proximidad, como Justo y Bueno, Éxito Express, Ara, Metro (Jumbo), junto a las tiendas de conveniencia Oxxo que ingresaron al país a partir del 2007, su penetración está lejos de naciones desarrolladas y otras en Latinoamérica.

Esta situación puede modificarse en el futuro en la medida en que se incremente la presencia de cadenas transnacionales en el país y vayan ganando cuota de mercado. El boletín de ventas al por menor que presentó el DANE para el 2015 recientemente es coherente con lo que ha venido pasando en el país desde los últimos años. Las ventas reales del comercio minorista presentaron un incremento de $4.5 \%$ respecto al mismo periodo de 2014 . Además, el personal ocupado promedio superó 5.3\% al del año anterior (Heraldo, 2015). Por otro lado, es importante especificar que los tenderos manejan un promedio de 15 proveedores, con un rango que oscila entre un mínimo de 4 y un máximo de 50 referencias en productos, siendo Bavaria (8.90\%), Coca-Cola (8.26\%), Alquería (6.47\%), Alpina (4.82\%), Protabaco (4.82\%) y Nutresa (4.60\%) los mayores influenciadores en las ventas de este tipo de negocio (El Tiempo, 2016). Hay otro aspecto por resaltar que es común ver en la actualidad: cómo las estanterías de estos establecimientos, que tradicionalmente estaban llenos de productos populares, ahora abren paso a marcas de valor y que antes eran exclusivos en las reconocidas cadenas.

En esta revisión de literatura acerca de la situación, existe un parentesco entre el estado de las mipymes de abarrotes en la economía de México y de Colombia. Es por esto que es pertinente para la investigación analizar a este tipo de organización, puesto que los aportes en este campo han demostrado que se trata de un integrante que se ha fortalecido en el curso de los años debido a factores culturales, económicos y comerciales. A causa de eso, la importancia del comercio abarrotero tanto a México y como a Colombia, aunque en términos netamente económicos y en su mayoría están ligados a la forma de comercialización y no al de la comunicación organizacional. Algunas investigaciones refieren las principales causas 
del impulso de este tipo de establecimientos de comercialización (no solo perteneciente a su creación sino también a su desarrollo y consolidación) y otros las fuentes primordiales de sus ventajas y es necesario preocuparse más y en forma efectiva por su desempeño en la economía de los dos países (Páramo, 2005; D’Andrea, Stengel y Goebel-Krstelj, 2003; D’Andrea, Lopez-Aleman y Stengel, 2006; Díaz, Lacayo y Salcedo, 2007).

\section{ESTADO DEL ARTE: INNOVACIÓN PARA EL DESARROLLO} SOCIOECONÓMICO DE LAS MIPYMES

La literatura revisada señala que la situación respecto a la innovación es un factor vital para el desarrollo de las microempresas donde el acceso a la tecnología y su implementación depende de las necesidades que tenga la empresa de acuerdo a su categoría. La tecnología es un componente fundamental que permite incrementar la eficiencia en toda clase de procesos como empresariales o de gestión (Ynzunza-Cortés, Izar-Landeta, y Ávila, 2013). Otra circunstancia, es que la mayoría de las mipymes latinoamericanas dependen de tecnología anticuada (Singh y Garg, 2008; Mejía, A., 2017) y, en ciertos casos específicamente tanto en México como en Colombia, no se otorga un papel explícito a la innovación, lo cual reduce su productividad (Pizzi y Brunet, 2013; Rodeiro-Pazos y López-Penabad, 2007).

La mayoría de las publicaciones en torno a que son más productivas y rentables las empresas que realizan I+D y la implementación de procesos tecnológicos e innovaciones son factores clave para el desarrollo de una microempresa, ya que este aspecto limita la competitividad de la misma (Mata y Woerter, 2013; Peña, 2018). También, hablan de la existencia de un escenario competitivo, turbulento y con cambiantes condiciones económicas y sociales, la empresa, mediante un proceso continuo de innovación de todas sus actividades, debe buscar ventajas competitivas sostenibles; enfocar la competitividad desde la cultura de la innovación muestra los valores y prácticas organizacionales, es decir, aquellas que estimulan la cohesión, el trabajo en equipo, el compromiso, la adaptabilidad, la flexibilidad, la aceptación del riesgo y la incertidumbre, generan importantes mejoras en el desempeño en términos de la calidad de los productos y servicios, en la eficiencia de los procesos internos, en las relaciones humanas internas, y en la relación de la empresa con su entorno inmediato (Gálvez y García, 2011; Crespi y Zuniga, 2012).Es así como el Manual de Oslo señala que innovar es introducir un nuevo o significativamente mejorado producto (bien o servicio) al mercado, o un nuevo proceso, método de comercialización o de organización, en el sistema de gestión mediante las prácticas internas de la empresa o en sus relaciones externas (Piening y Salge, 2015). 
A pesar que los microempresarios reconocen tanto en México como en Colombia como una ventaja la incorporación de la innovación y mejora de la calidad de sus productos, lo que les permitiría competir y permanecer en el mercado (Reyes, Parrales, Pionce y Soledisma, 2019). Para las mipymes frecuentemente es complicado y costoso financiar proyectos de innovación a través de fuentes bancarias debido a factores como la complejidad de la información y la posible pérdida de autonomía administrativa (Solís, 2013; Monge y Rodríguez, 2012), por ello, generalmente deben limitarse a fuentes propias.

Otra dirección en la que se enfoca la innovación para este tipo de organizaciones como son las tiendas de abarrotes, distinta a la administración o al management en las que se encontraron publicaciones desde una perspectiva más sociológica en la que realiza una crítica a la ideología gestionaria donde poco abona a la comprensión de las especificidades de las mipymes, obligadas a asumir modelos, y sugerencias que por un lado invisibilizan muchas de las características más importantes de este tipo de empresas, y por otro lado, establecen un deber ser que se traduce en formas de exclusión social (Saldaña, 2014; Molina, López, y Contreras, 2014).

Por último, es interesante observar la dirección de las publicaciones que están enfocadas sobre la innovación en su producto o gestión, pero muy pocas lo dirigen a la comunicación en microorganizaciones, por lo tanto, la ideología gestionaría donde poco abona a la comprensión de las especificidades de las mipymes, obligadas a asumir modelos y sugerencias que por un lado invisibilizan muchas de las características más importantes de este tipo de empresas (Saldaña, 2014; Molina, López, y Contreras, 2014). Sin embargo, gran parte del éxito tiene mucho que ver con la cultura organizacional y su predisposición a innovar en las mipymes. Además, la importancia de la comunicación organizacional, como la plataforma de la generación, divulgación y apropiación del conocimiento para innovar, a través de nuevas plataformas y nuevos métodos para establecer relaciones más claras, transparentes y directas, ayudando a mejorar en todos los aspectos a este tipo de organizaciones.

\section{MARCO TEÓRICO: GESTIÓN DE LA IDENTIDAD}

\section{EN MICROORGANIZACIONES COMO LAS TIENDAS DE ABARROTES}

En relación con el desarrollo de investigaciones empíricas acerca de la gestión de la identidad en el sector de las tiendas de abarrotes, siendo entendida como la forma de desarrollar y controlar la identidad a través de las actividades que la organización desarrolla deliberadamente para sus stakebolders, a través de una comunicación coherente que exprese la identidad de la empresa con hechos comprobables (Takaki,Bravo y Martínez, 2014).No obstante, las tiendas 
muestran el trasfondo cultural que devela su carácter auténticamente social, y en tanto que tal son inseparables de su comunidad, de su vecindario, refuerzan su identidad (Páramo, 2009).

En muchos de los trabajos empíricos se suele optar por simplificar las dimensiones de análisis, debido que, dependiendo de la perspectiva de estudio, puede resultar poco adecuado investigar la percepción de ciertas dimensiones (Takaki et al., 2014). Sin embargo, se señalan diversos aspectos como la cultura, el comportamiento, la comunicación, la identidad visual, la estrategia, la estructura, las condiciones de mercado, la personalidad de marca, la orientación del empleado al cliente o las iniciativas de recursos humanos (Coleman, de Chernatony y Christodoulides, 2011). Entre otros se exponen dentro de las dimensiones de la identidad como la actual, la comunicada, la concebida, la convenida, la cultural, la ideal y la deseada (Balmer, 2012). Pero este tipo de dimensiones no se han aclarado en su totalidad, lleguen a corresponder a las características propias de microorganizaciones como las tiendas de abarrotes en países como México y Colombia.

De manera general, en las pocas investigaciones identificadas en la literatura se pudo observar la existencia de propuestas de estudio sobre la gestión de la identidad en microorganizaciones que permitan aplacar acciones de mejoramiento, siendo dentro de la comunicación organizacional, la identidad de la empresa entendida como una parte estratégica y, además de estar alineada con el resto de actividades que desarrolla la empresa, debe servir como fuente de diferenciación. Asimismo, las dimensiones de los atributos que componen la identidad de una empresa parten de tres ejes estructurales que la definen así: el primer eje es el de la historia de la organización, la trayectoria recorrida desde la fundación de la empresa hasta su presente; el segundo eje es el proyecto optado por la empresa en su presente para satisfacer sus metas; el tercer eje es la cultura de la organización que no está anclado temporalmente ni al presente ni al pasado, sino de forma simultánea (Villafañe, 1999, p. 18).

En este sentido, la versión más reciente es el AC4ID test de Balmer y Soenen (2005), donde proponen siete tipos de identidad dentro de la constelación de la marca corporativa: la identidad actual o real representa lo que es la compañía ; la identidad comunicada es la marca corporativa que la empresa comunica; la identidad concebida es la percepción de la marca por parte de los grupos de interés; la identidad convenida es lo que la marca corporativa promete ser; la identidad cultural son los valores internos de la marca; la identidad ideal, lo que la marca debería ser; la identidad deseada es la marca corporativa deseada para la empresa.

Por lo tanto, de acuerdo con estas ideas, para comprender la identidad de una microorganización es necesario profundizar en otras investigaciones el carácter complejo y multidisciplinar de la identidad, con la finalidad de gestionar cada uno de estos elementos que sean propios de este tipo de organización, por medio de modelos de innovación en comunicación de la manera más adecuada. En otras palabras, la gestión de la identidad a través de sus dife- 
rentes dimensiones es el antecedente principal dentro de los procesos comunicacionales al ser el epicentro que permite al interior de una organización acelerar o detener los procesos de innovación.

\section{PLANTEAMIENTO DE HIPÓTESIS}

De acuerdo con la información presentada con anterioridad, se realizó un análisis correlacional para conocer el nivel de significancia entre las variables. A efectos de explicar la asociación entre las variables innovación y las variables que conforman el constructo de identidad se utilizó la prueba de chi-cuadrado. De esta forma fue posible plantear las siguientes hipótesis de investigación.

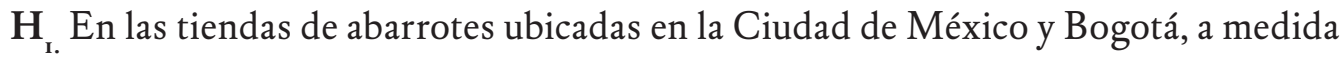
que éstas son capaces de destacar su innovación en productos, ello tiene una influencia positiva en la gestión de la identidad de estas tiendas.

$\mathbf{H}_{2 .}$ En las tiendas de abarrotes ubicadas en la Ciudad de México y Bogotá, a medida que éstas son capaces de destacar su innovación en procesos, ello tiene una influencia positiva en la gestión de la identidad de estas tiendas.

$\mathbf{H}_{3 .}$ En las tiendas de abarrotes ubicadas en la Ciudad de México y Bogotá, a medida que éstas son capaces de destacar su innovación en gestión, ello tiene una influencia positiva en la gestión de la identidad de estas tiendas.

\section{METODOLOGíA DE LA INVESTIGACIÓN}

En este apartado se explican aspectos metodológicos sobre la configuración de la muestra y el proceso de recogida de información y la justificación de las variables.

\section{Muestra}

La muestra utilizada es de 200 tiendas de abarrotes ubicadas en los países de México, en la Ciudad de México con 99 negocios, y Colombia, en la ciudad de Bogotá con 101 negocios. La información se recogió aplicando un cuestionario estructurado dirigido al propietario de la tienda. El trabajo de campo se realizó entre los meses de julio y septiembre de 2018. La preci- 
sión de la muestra final del total de países se caracteriza por un error máximo de cinco puntos porcentuales con un nivel de confianza de $95 \%$. Todas las tiendas estaban en barrios con características similares en sus países, lo cual denota el papel del entorno y otros factores para hallar a las diferencias que pueden llegar a ser el foco de la examinación al descubrir por qué los casos son diferentes al revelar la estructura subyacente que genera o permite tal variación.

\section{Medición de variables}

A efectos de explicar las variables utilizadas en este apartado se exponen las medidas de innovación, de identidad y las variables de control.

\section{Innovación}

Para la investigación se utilizaron como indicadores para medir la innovación, se determinó seguir los trabajos previos de Piening y Salge (2015); Astudillo y Briozzo (2016) y García-Pérez (2016), quienes adoptaron la clasificación propuesta proveniente de la tercera edición del Manual de Oslo de la OCDE (2005), en las dimensiones de producto, proceso y gestión; asimismo, se utilizó para su medición una escala Likert de 5 puntos adaptada para las mipymes, donde ( 1 = totalmente en desacuerdo, $5=$ totalmente de acuerdo). Las dimensiones de la innovación en productos se conforman con la media de dos factores: los cambios o mejoras en productos o servicios existentes y la comercialización de nuevos productos/ servicios. La dimensión innovación en procesos se conforma con la media de dos factores: los cambios o mejoras en los procesos productivos y la adquisición de nuevos equipos. Por último, la variable innovación en gestión se conforma con la media aritmética de tres factores: el sistema de gestión y dirección, el sistema de compras y aprovisionamientos y el sistema comercial y ventas. Para comprobar la validez de estas medidas se determinó el estadístico alpha de Cronbach, donde los valores superiores a 0,711 señalan la validez de las escalas utilizadas.

\section{Identidad}

En este estudio se utilizaron como indicadores para medir la identidad de microempresas como las tiendas de abarrotes para medir las dimensiones, se preguntó al dueño de la tienda sobre personalidad corporativa a partir de trabajos previos de Villafañe (1999), Christensen y Askegaard (2001) y el mix cultural de identidad corporativa utilizada en los trabajos de Zarebska (2010) y Balmer (2012), entre otros. Para esto se usó una escala Likert de 1 a 5 , donde 1 = totalmente en desacuerdo y 5 = totalmente de acuerdo. Asimismo, para com- 
probar la validez de estas medidas se determinó el estadístico alpba de Cronbach, donde los valores superiores a 0,721 señalan la validez de las escalas utilizadas.

\section{Variables de control}

Como variables de control se han considerado el género, la edad del tendero y la antigüedad por el número de años transcurridos desde el inicio de actividad de la mipyme.

\section{RESULTADOS Y ANÁLISIS}

El análisis y la discusión de los resultados se estructura en dos partes. En primer lugar se analizan los resultados descriptivos y en segundo lugar los análisis inferenciales a partir de los modelos de investigación planteados.

\section{Resultados descriptivos}

De los 201 encuestados, como puede observarse en el Cuadro 1, en la muestra total de las dos ciudades no existe una diferencia significativa, los hombres representan $51 \%$, seguidos por las mujeres con $49 \%$. Sin embargo, en la Ciudad de México hay un predominio de mujeres $(52,5 \%)$ y de hombres $(47,5 \%)$; en contraste con la ciudad de Bogotá, donde existe un predominio de hombres $(54,5 \%)$ y de mujeres $(45,5 \%)$ que manejan las tiendas. Asimismo, en la muestra se aprecia que el rango de edad del tendero más común es el que va de los cuarenta a los cuarenta y nueve años $(25,5 \%)$. No obstante, observamos una diferencia significativa en el rango de edad que oscila entre los veinte y veintinueve años, siendo menor el número de tenderos con este rango en la Ciudad de México $(8,1 \%)$ y predomina más las personas jóvenes en esta labor en la ciudad de Bogotá (20,8\%).

La antigüedad de la tienda en la muestra consolidada se observa en su tipo de operación, la cual está entre los 6 a 15 años (44\%). Destaca, de manera visible, que casi una quinta parte de las tiendas oscilan entre 16 a 25 años (13,0\%), y negocios con más de 25 años (4,5\%). Al contrastar estos resultados entre las ciudades, encontramos que en la Ciudad de México es destacable que tres cuartas partes de las tiendas están en los dos primeros grupos ( 1 a 5 y 6 a 15 años). La diferencia está en que más de una cuarta parte de los establecimientos en la Ciudad de México (26,3\%) tienen un sostenimiento en el tiempo de este tipo de empresas mayores a 16 años de antigüedad, comparado con la ciudad de Bogotá $(8,9 \%)$. 


\section{Resultados inferenciales}

Las estimaciones que se presentan en el Cuadro 1 se realizaron a partir de correlaciones de chi-cuadrado. A continuación, se describen los resultados de cada una de las relaciones analizadas.

CUADRO 1. RESULTADOS DE LA CHI-CUADRADO ENTRE LAS VARIABLES DE INNOVACIÓN E IDENTIDAD

\begin{tabular}{|c|c|}
\hline \multicolumn{2}{|l|}{ RESULTADOS DE MÉXICO } \\
\hline CORRELACIÓN DE VARIABLES & CHI-CUADRADO DE PEARSON \\
\hline $\begin{array}{l}\text { Innovación en productos - Cambios y mejoras e Identidad } \\
\text { deseada corporativa }\end{array}$ & $(\chi 2=30,525 ; g l=1 ; \alpha=0,000)$ \\
\hline $\begin{array}{l}\text { Innovación en productos - Cambios y mejoras e Identidad } \\
\text { convenida }\end{array}$ & $(\chi 2=21,536 ; g l=1 ; \alpha=0,000)$ \\
\hline $\begin{array}{l}\text { Innovación en productos - Adquisición de nuevos equipos e } \\
\text { Identidad convenida }\end{array}$ & $(\chi 2=19,613 ; g \mid=1 ; \alpha=0,000)$ \\
\hline $\begin{array}{l}\text { Innovación en productos - Cambios y mejoras e Identidad } \\
\text { promocional comunicada }\end{array}$ & $(\chi 2=17,839 ; g l=1 ; \alpha=0,000)$ \\
\hline $\begin{array}{l}\text { Innovación en productos - Cambios y mejoras e Identidad } \\
\text { sectorial }\end{array}$ & $(\chi 2=17,600 ; g l=1 ; \alpha=0,000)$ \\
\hline \multicolumn{2}{|c|}{ RESULTADOS CONSOLIDADOS DE COLOMBIA } \\
\hline CORRELACIÓN DE VARIABLES & CHI-CUADRADO DE PEARSON \\
\hline $\begin{array}{l}\text { Innovación en productos - Cambios y mejoras e Identidad } \\
\text { ideal corporativa }\end{array}$ & $(\chi 2=9,942 ; g l=1 ; \alpha=0,002)$ \\
\hline $\begin{array}{l}\text { Innovación en gestión - Sistemas de gestión y Dirección e } \\
\text { Identidad visual comunicada }\end{array}$ & $(\chi 2=8,436 ; g l=1 ; \alpha=0,004)$ \\
\hline $\begin{array}{l}\text { Innovación en productos - Nuevos productos y Servicios e } \\
\text { Identidad deseada corporativa }\end{array}$ & $(\chi 2=8,204 ; g l=1 ; \alpha=0,004)$ \\
\hline $\begin{array}{l}\text { Innovación en productos - Cambios y mejoras e Identidad } \\
\text { cultural }\end{array}$ & $(\chi 2=7,430 ; g l=1 ; \alpha=0,006)$ \\
\hline $\begin{array}{l}\text { Innovación en productos - Cambios y mejoras e Identidad } \\
\text { deseada corporativa }\end{array}$ & $(\chi 2=6,773 ; g l=1 ; \alpha=0,009)$ \\
\hline
\end{tabular}


CUADRO 2. RESULTADOS CONSOLIDADOS DE MÉXICO Y COLOMBIA

\begin{tabular}{|l|l|}
\hline \multicolumn{1}{|c|}{ CORRELACIÓN DE VARIABLES } & \multicolumn{1}{c|}{ CHI-CUADRADO DE PEARSON } \\
\hline $\begin{array}{l}\text { Innovación en productos - Cambios y mejoras e Identidad } \\
\text { deseada corporativa }\end{array}$ & $(\chi 2=44,917 ; g l=4 ; \alpha=0,000)$ \\
\hline $\begin{array}{l}\text { Innovación en gestión - Sistemas de gestión y Dirección e } \\
\text { Identidad promocional comunicada }\end{array}$ & $(\chi 2=23,112 ; g \mid=4 ; \alpha=0,000)$ \\
\hline $\begin{array}{l}\text { Innovación en gestión - Sistemas de compras y } \\
\text { Aprovisionamiento e Identidad promocional comunicada }\end{array}$ & $(\chi 2=21,409 ; g l=4 ; \alpha=0,000)$ \\
\hline $\begin{array}{l}\text { Innovación en productos - Nuevos productos y Serviciose } \\
\text { Identidad deseada corporativa }\end{array}$ & $(\chi 2=22,181 ; g l=4 ; \alpha=0,000)$ \\
\hline $\begin{array}{l}\text { Innovación en productos - Adquisición de nuevos equipos e } \\
\text { Identidad deseada corporativa }\end{array}$ & $(\chi 2=22,049 ; g l=4 ; \alpha=0,000)$ \\
\hline
\end{tabular}

FUENTE: ELABORACIÓN PROPIA.

\section{Efecto de la innovación sobre identidad en las tiendas de abarrotes}

En el Cuadro 2 se recogen los resultados de las relaciones más significativas entre los dos constructos. Se evidencia que en el resultado consolidado de México y Colombia tiene una relación significativa entre innovación en productos mediante el factor de cambios y mejoras en el establecimiento y la identidad deseada corporativa $(\chi 2=44,917 ; \mathrm{gl}=4 ; \alpha=0,000)$, lo cual indica que cuanto mayor es la actividad innovadora en productos/servicios, mayor es el valor en la visión del negocio, lo que también se aprecia particularmente en el caso de México ( $\chi 2=30,525$; gl=1; $\alpha=0,000)$; sin embargo, en Bogotá es mucho menor su significancia $(\chi 2=6,773 ; \mathrm{gl}=1 ; \alpha=0,009)$. En cuanto a la innovación en la gestión, en los datos consolidados también se observa a nivel de la tienda con una influencia positiva y significativa con la identidad promocional corporativa $(\chi 2=23,112 ; \mathrm{gl}=4 ; \alpha=0,000)$, lo que implica que cuanta más innovación en los sistemas de gestión y dirección desarrollen este tipo de mipymes mediante nuevos procedimientos para sistematizar ciertas operaciones (compras, control de calidad, compras, etc.), mayor será el valor para promover el negocio a sus públicos; esto se verifica igualmente en Colombia $(\chi 2=8,436 ; \mathrm{gl}=1 ; \alpha=0,004)$ y en México no resultó significativa. Finalmente, respecto a la innovación en productos, se aprecia también su efecto positivo y significativo en el consolidado con factores como el 
de nuevos productos y servicios $(\chi 2=22,181 ; \mathrm{gl}=4 ; \alpha=0,000)$ y la adquisición de nuevos equipos $(\chi 2=22,049 ; \mathrm{gl}=4 ; \alpha=0,000)$; esto señala que la innovación en productos tiene un impacto favorable en la identidad deseada de la tienda de abarrotes, al tener una imagen sobre lo que quieren ser y cómo llegar a serlo. Este tipo de innovación en México, la adquisición de nuevos equipos de la tienda $(\chi 2=19,613 ; \mathrm{gl}=1 ; \alpha=0,000)$ tiene mayor significancia al comparar con Colombia $(\chi 2=6,773 ; \mathrm{gl}=1 ; \alpha=0,009)$; de manera que la relación de la innovación en productos en Colombia mediante el factor de cambios y mejoras es mayor con la identidad cultural, influyendo en el pensamiento, valores y creencias de la tienda de abarrotes $(\chi 2=7,430 ; \mathrm{gl}=1 ; \alpha=0,006)$.

Los resultados presentados permiten comprobar las hipótesis planteadas para este trabajo con sus respectivas diferencias en cada uno de los países. En cuanto a las variables de control, los resultados muestran que para el género, la edad del tendero y la antigüedad no resultó significativa; para esta relación, de manera individual, estos resultados son verificables para México y Colombia.

\section{CONCLUSIONES}

La problemática de las tiendas de abarrotes como una microorganización empresarial y la innovación se perciben como temas estratégicos para el éxito del negocio, dadas las tendencias competitivas presentadas en el mercado; de ahí la importancia de estudiar las relaciones entre los diferentes tipos de innovación y la identidad en este tipo de mipymes. Los resultados de esta investigación han demostrado que la actividad innovadora de la tienda de abarrotes ejerce una influencia positiva y significativa sobre los factores que constituyen la identidad, con la excepción de la innovación en procesos donde no se obtuvieron datos significativos sobre alguno de los factores de la identidad. En relación con el impacto positivo de la actividad innovadora en productos y sistemas de gestión se verifican las hipótesis 1 y 3 , y estos resultados aportan a las investigaciones previamente realizadas como los de Pizzi y Brunet (2013); Mata y Woerter (2013). En cuanto a la innovación en procesos, los resultados confirman solo en México un efecto positivo en la identidad comunicada, la identidad sectorial y la identidad deseada, pero no se halló mayor significancia en Colombia como en el consolidado, por lo que solo se confirma parcialmente la hipótesis 2 del trabajo de investigación.

Esta investigación sugiere líneas de investigación futuras dadas sus limitaciones. En primer lugar, la muestra utilizada por país es pequeña, por lo que a dicho nivel los resultados se deben reservar su interpretación; una muestra más amplia permitirá profundizar en 
cada nación sobre sus particularidades específicas con el fin de promover programas más ajustados a ellas. En segundo lugar, es necesario profundizar en el hallazgo del impacto de la innovación en la identidad corporativa en las tiendas de abarrotes, pues es un tema muy relevante para estudiar, y en la literatura no existen muchos estudios entre estos dos constructos en microorganizaciones; en este sentido resultará muy útil realizar más estudios específicos más detallados sobre la cuestión.

\section{REFERENCIAS}

Argenti, P. (2014). Comunicación estratégica y su contribución a la reputación. Madrid: Lid Editorial. Astudillo, S. y Briozzo, A. (2016). Innovación en las mipymes manufactureras de Ecuador y Argentina. Semestre Económico, 19(40), pp. 117-144. https://doi.org/10.22395/seec.v19n40a5

Balmer, J.M.T. (2012). Corporate Brand Management Imperatives: Custodianship, Credibility and Calibration. California Management Review, Vol. 54, núm. 3, pp. 6-33. https://doi. org/10.1525/cmr.2012.54.3.6

Bedoya, C. y Jaramillo López, A. (2014). Las tiendas de conveniencia, una nueva opción para los consumidores en la ciudad de Medellín. Descripción del formato de conveniencia y los factores que lo representan en el contexto de la ciudad de Medellín. Publicidad: revista latinoamericana de publicidad, Vol. 2, núm. 1, pp. 106-123. Recuperado de https://revistas.upb.edu.co/index.php/ publicuidad/article/view/2806/2449 https://doi.org/10.24275/uami.hq37vn60x

Bolívar, C. (2012). La comunicación integral en las Pymes. Recuperado de www.gestiopolis.com/ canales/derrhh/articulos/65/cb/cipymes.pdf.

Coleman, D., De Chernatony, L. y Christodoulides, G. (2011). B2B service brand identity: Scale development and validation. Industrial Marketing Management, 40(7), pp. 1063-1071. https:// doi.org/10.1016/j.indmarman.2011.09.010

Crespi, G. y Zuniga, P. (2012). Innovation and productivity: evidence from six Latin American countries. World development, 40(2), pp. 273-290. https://doi.org/10.1016/j.worlddev.201 1.07.010

Christensen, L.T. y Askegaard, S. (2001). Corporate identity and corporate image revisited - A semiotic perspective. European Fournal of Marketing, 35(3/4), pp. 292-315. https://doi. org/10.1108/03090560110381814

D’Andrea, G., López-Alemán, B. y Stengel, A. (2006). Why small retailers endure in Latin America. International Fournal of Retaily Distribution Management, 34 (9), pp. 661-673. https://doi. org/10.1108/09590550610683184

D’Andrea, G., Stengel, E.A. y Goebel-Krstelj, A. (2003). Crear valor para los consumidores emergentes. Harvard Business Review América Latina, pp. 105-112.

Díaz, A., Lacayo, J. y Salcedo, L. (2007) Cómo vender a las tiendas de barrio en América Latina. The McKinsey Quarterly, edición especial, pp. 81-93. 
El Economista. (2016). Sector abarrotero crece 4.3\% en el primer semestre. Recuperado de http:// eleconomista.com.mx/industrias/2014/08/19/sector-abarrotero-crece-43-primer-semestre

El Heraldo Colombia. (2015). Tiendas de barrio, modelo exitoso que compite contra 'gigantes'. Recuperado de http://www.elheraldo.co/economia/tiendas-de-barrio-modelo-exitoso-que-compite-contra-gigantes-196520 https://doi.org/10.22209/mensi.n7a18

FUNDES. (2008). Dinámica de la empresa familiar Pyme. Estudio exploratorio en Colombia. Colombia.

Gálvez Albarracín, E. J. y García Pérez de Lema, D. (2011). Cultura organizacional y rendimiento de las MiPymes de mediana y alta tecnología: un estudio empírico en Cali, Colombia. Cuadernos de Administración, 24(42), pp. 125-145. Recuperado de http://www.scielo.org.co/scielo. php?script=sci_arttextypid=S0120-35922011000100006ylng=enytlng=es https://doi. org/10.25100/cdea.v30i51.44

García-Pérez, D.L. (2016). Efecto de la innovación en el crecimiento y el desempeño de las mipymes de la alianza del pacífico. un estudio empírico. Estudios Gerenciales, 32(141), pp. 326-335. https://doi.org/10.1016/j.estger.2016.07.003

Giraldo,J.(2002).El comercio en la Economía de Colombia. Recuperado dehttp://www.gestiopolis.com/ el-comercio-en-la-economia-de-colombia https://doi.org/10.31819/9783954879304-001

López Juárez, P.; López Hernández, I. y Martínez Hernández, M. (2015). Ventaja competitiva de las tiendas de proximidad. Raxión, Año 2, Núm. 2, Enero-Abril, 2015. Universidad Tecnológica de León, pp.1-7. Recuperado de http://reaxion.utleon.edu.mx/Art_Ventaja_Competitiva_Tiendas_de_Proximidad.html https://doi.org/10.14483/2248762x.8647

Lopez Salazar, A. y Contreras, Soto. (2010). Estrategias empresariales en la economía basada en el conocimiento, SINNCO 2010, La responsabilidad social empresarial del sector alimenticio. Recuperado de https://www.researchgate.net/publication/259758721_Lopez_y_ Contreras_Estrategias_Empresariales_en_la_Economia_Basada_en_el_Conocimiento_ SINNCO_2010_La_Responsabilidad_Social_Empresarial_del_sector_alimenticio/citation/ download https://doi.org/10.32468/dtseru.72

Martín, R.(2017). Tienditas en riesgo.ElEconomista. Recuperado de http://eleconomista.com.mx/ antipolitica/2014/01/03/tienditas-riesgo

Mata, J. y Woerter, M. (2013). Risky innovation: The impact of internal and external RyD strategies upon the distribution of returns. Research Policy, 42(2), pp. 495-501. https://doi.org/ 10.10164respol.2012.08.004

Molina, R.; López, A. y Contreras, R. (2014). El emprendimiento y crecimiento de las Pymes. Acta Universitaria, vol. 24, núm. 1, pp. 59-72. Recuperado de http://www.redalyc.org/articulo. oa?id=41648308006

Monge, R. y Rodríguez, J. A. (2012). El impacto de los servicios financieros y de capacitación en las MiPyME de Costa Rica. Banco Interamericano de Desarrollo, División de Mercados de Capital e Instituciones Financieras (IFD/CMF). Washington. Banco Interamericano de Desarrollo, BID. https://doi.org/10.18235/0001907

Organización para la Cooperación y el Desarrollo Económicos. (2005). Manual de Oslo: Guía para la recogida e interpretación de datos sobre innovación. París: OCDE. https://doi.or$\mathrm{g} / 10.1787 / 9789264065659$-es 
Páramo, D. (2009). Le commerce traditionnel colombien: un espace de renforcement culturel pour les consommateurs [Thèse doctorale n. ${ }^{\circ}$ 705]. Faculté de Sciences Economiques et Sociales, Université de Genève (Suisse).

Páramo Morales, D. (2012). Tiendas de barrio en Colombia. Pensamiento y Gestión, (32), pp. 1-5. Recuperado de http://www.scielo.org.co/scielo.php?script=sci_arttextypid=S1657$62762012000100001 \mathrm{ylng}=$ enytlng=es

Páramo, D. (2005). Valores, creencias y orientación temporal del consumidor de tiendas de barrio de Barranquilla. Encuentro Nacional de Investigación en Administración, pp. 83-110.

Pizzi, A. y Brunet, I. (2013). Creación de empresas, modelos de innovación y pymes. Cuadernos del CENDES (83), pp. 53-74.

Portafolio. (2016). La fortuna en las tiendas de barrio. Portafolio.co. Recuperado de https://www. portafolio.co/opinion/otros-columnistas-1/fortuna-tiendas-barrio-496805 https://doi.org/ 10.22209/mensi.n7a18

Reyes, J. E. P.; Parrales, D. A. N.; Ponce, D. K. P.; Pionce, M. S. P. y Soledispa, M. L. F. (2019). Innovación tecnológica para el desarrollo socio económico de MIPYMES de la zona sur de Manabí. Revista Ibérica De Sistemas e Tecnologias De Informação, pp. 221-233. Recuperado de https://search.proquest.com/docview/2258687256?accountid $=41021$

Rodeiro-Pazos, D. y López-Penabad, M. (2007). La innovación como factor clave en la competitividad empresarial: un estudio empírico en pymes. Revista Galega de Economía, 16(2), pp. 1-18.

Saldaña Rosas, A. (2014). Integración regional y sistemas locales de innovación: desafíos para las MIPYMES. Una perspectiva desde México. Espacio Abierto, 23, pp. 629-642. Recuperado de http://www.redalyc.org/articulo.oa?id=12232821004

Solís, K. (2013). Financiamiento de la banca comercial a micro, pequeñas y medianas empresas en México. Problemas del Desarrollo. Revista Latinoamericana de Economía, 44 (172), pp. 160 162. https://doi.org/10.18356/2f365699-es

Soria Romo, R. (2008). Comunicación Organizacional: Un modelo aplicable a la microempresa. TEACS, año 1, núm. 1.

Suvatjis, J. Y., De Chernatony, L. y Halikias, J. (2012). Assessing the six-stationcorporate identity model: A polymorphic model. Fournal of Product y BrandManagement, 21(3), pp. 153-166. https://doi.org/10.1108/10610421211228775

Takaki, M.; Bravo, R. y Martínez, E. (2014). La gestión de la identidad corporativa en la Universidad: análisis y consecuencias desde la perspectiva del profesorado. Revista Europea de Dirección y Economía de la Empresa. Facultad de Economía y Empresa. Universidad de Zaragoza, España. https://doi.org/10.1016/j.redee.2014.05.001

Tiempo, C. (2016). Las tiendas siguen 'mandando' en el comercio. portafolio.co. Recuperado de http://www.portafolio.co/economia/finanzas/tiendas-siguen-mandando-comercio-28326

Villagra, N. y López, B. (2013). Analysis of values and communication of the Responsible Brands. Corporate Brand strategies for sustainability. Communication y Society / Comunicación y Sociedad, 26(1), pp. 201-226. 
Dinero.com. (2014). La tienda, el aliado de la casa. Dinero.com. Recuperado de http://www.dinero.com/edicion-impresa/informe-especial/articulo/tiendas-barrio-colombia/201683 https:// doi.org/10.29057/icshu.v3i5.944

INEGI. (2015). Censos Económicos 2014. Recuperado de http://www.inegi.org.mx/saladeprensa/ boletines/2015/especiales/especiales2015_12_38.pdf

Ynzunza-Cortés; Izar-Landeta y Ávila Acosta. (2013). Recursos y Capacidades de Tecnología y Desempeño. Conciencia Tecnológica, núm. 46, pp. 36-42. Instituto Tecnológico de Aguascalientes, México.

Zarebska, A. (2010). Practical problems of managing corporate identity of capital structures in Poland. The International Business y Economics Research Fournal, 9(1), pp. 9-15. https://doi. org/10.19030/iber.v9i1.504 